\title{
Unfounded Sets for Disjunctive Hybrid MKNF Knowledge Bases
}

\author{
Spencer Killen and Jia-Huai You \\ Department of Computing Science, University of Alberta \\ \{sjkillen, jyou\}@ualberta.ca
}

\begin{abstract}
Combining the closed-world reasoning of answer set programming (ASP) with the open-world reasoning of ontologies broadens the space of applications of reasoners. Disjunctive hybrid MKNF knowledge bases succinctly extend ASP and in some cases without increasing the complexity of reasoning tasks. However, in many cases, solver development is lagging behind. As the result, the only known method of solving disjunctive hybrid MKNF knowledge bases is based on guess-and-verify, as formulated by Motik and Rosati in their original work. A main obstacle is understanding how constraint propagation may be performed by a solver, which, in the context of ASP, centers around the computation of unfounded atoms, the atoms that are false given a partial interpretation. In this work, we build towards improving solvers for hybrid MKNF knowledge bases with disjunctive rules: We formalize a notion of unfounded sets for these knowledge bases, identify lower complexity bounds, and demonstrate how we might integrate these developments into a DPLLbased solver. We discuss challenges introduced by ontologies that are not present in the development of solvers for disjunctive logic programs, which warrant some deviations from traditional definitions of unfounded sets. We compare our work with prior definitions of unfounded sets.
\end{abstract}

\section{Introduction}

Minimal Knowledge and Negation as Failure (MKNF) is a modal autoepistemic logic defined by Lifschitz (1991) which extends first-order logic with two modal operators $\mathbf{K}$ and not . It was later built upon by Motik and Rosati (2010) to define hybrid MKNF knowledge bases, where rule-based MKNF formulas along with a description logic (DL) knowledge base intuitively encapsulate the combined semantics of answer set programs and ontologies. One argument for using hybrid MKNF is the existence of a proof theory based on guess-and-verify - one can enumerate partitions (a term that corresponds to interpretation in first-order logic) and for each one check whether it is an MKNF model. Such an approach is not efficient enough to be practical.

To address the above issue, Ji et al. (2017) give a definition of unfounded sets and an abstract DPLL-based solver (Nieuwenhuis, Oliveras, and Tinelli 2006) for normal hybrid MKNF knowledge bases, where rules are constrained to a single atom in the head.
Disjunctive rules are a powerful extension to answer set programming that increases the expressive power of programs in the polynomial complexity hierarchy (Eiter and Gottlob 1995). In this work, we extend the work of Ji et al. (2017) by defining unfounded sets for disjunctive hybrid MKNF knowledge bases and we investigate the properties of such sets. This task turns out to be substantially more challenging than the normal case. We show the following main results. First, we show that the problem of determining whether an atom is unfounded w.r.t. a given (partial) partition is coNP-hard. This result is somewhat surprising in that the claim holds even for normal rules under the condition that the entailment relation in the underlying DL is polynomial. This shows that the polynomial construction of the greatest unfounded set as given by Ji et al. (2017) for the normal case is only an approximation. Our proof relies on an encoding that takes care of several conditions simultaneously (the hardness in the presence of non-disjunctive rules and the entailment relation assuming the DL is polynomial). Then, we formulate a polynomial operator to approximate the greatest unfounded set of disjunctive hybrid MKNF knowledge bases. Unlike the conventional definition of unfounded sets for disjunctive logic program (Leone, Rullo, and Scarcello 1997), greatest unfounded sets under our definition exist unconditionally. We identify the conditions under which our approximation becomes exact for normal as well as for disjunctive hybrid MKNF knowledge bases. These conditions are also the ones under which the coNP-hardness reduces to polynomial complexity for the normal and disjunctive cases respectively, thus these results pinpoint the sources that contribute to the hardness of computing greatest unfounded sets in general. Finally, based on these results, we formulate a DPLL-based solver, where the computation of unfounded sets becomes a process of constraint propagation for search space pruning.

The next section provides preliminaries. Section 3 gives the definition of unfounded sets and studies its properties. Section 4 shows the main technical results concerning the challenges of computing unfounded sets, which lead to a formulation of a DPLL-based solver in Section 5. Section 6 discusses related work. We make concluding remarks in Section 7. 


\section{Preliminaries}

Minimal knowledge and negation as failure (MKNF) extends first-order logic with two modal operators, $\mathbf{K}$ and not, for minimal knowledge and negation as failure respectively. MKNF formulas are constructed from first-order formulas using these two modal operators for closed-world reasoning. Intuitively, $\mathbf{K} \psi$ asks whether $\psi$ is known w.r.t. a collection of "possible worlds" - the larger the set, the fewer facts are known - while not $\psi$ checks whether $\psi$ is not known, based on negation as failure. An MKNF structure is a triple $(I, M, N)$ where $I$ is a first-order interpretation and $M$ and $N$ are sets of first-order interpretations. Operators shared with first-order logic are defined as usual. An $M K N F$ interpretation $M$ is a set of first-order interpretations ("possible worlds"). Hybrid MKNF knowledge bases rely on the standard name assumption (Motik and Rosati 2010). Under this assumption, every first-order interpretation in an MKNF interpretation is required to be a Herbrand interpretation with a countably infinite number of additional constants. We refer to these constants as names. The satisfiability relation under an MKNF structure is defined as:

- $(I, M, N) \models A$ if $A$ is true in $I$ where $A$ is a first-order atom

- $(I, M, N) \models \neg F$ if $(I, M, N) \not \models F$

- $(I, M, N) \models F \wedge G$ if $(I, M, N) \models F$ and $(I, M, N) \models$ $G$

- $(I, M, N) \models \exists x, F$ if $(I, M, N) \models F[\alpha / x]$ for some name $\alpha$ (where $F[\alpha / x]$ is obtained by replacing every occurrence of the variable $x$ with $\alpha$ )

- $(I, M, N) \models \mathbf{K} F$ if $(J, M, N) \mid=F$ for each $J \in M$

- $(I, M, N) \models \operatorname{not} F$ if $(J, M, N) \not \models F$ for some $J \in N$

Other symbols such as $\vee, \forall$, and $\supset$ are interpreted in MKNF as they are in first-order logic. An MKNF interpretation $M$ satisfies a formula $F$, written $M \models_{M K N F} F$, if $(I, M, M) \models F$ for each $I \in M$.

Definition 1. An MKNF model $M$ of a formula $F$ is an $M K N F$ interpretation such that $M \models_{M K N F} F$ and there does not exist an MKNF interpretation $M^{\prime} \supset M$ such that $\left(I, M^{\prime}, M\right) \models F$ for each $I \in M^{\prime}$.

Following Motik and Rosati (2010), a hybrid MKNF knowledge base $\mathcal{K}=(\mathcal{O}, \mathcal{P})$ consists of a decidable description logic (DL) knowledge base $\mathcal{O}$ (typically called an ontology) which is translatable to first-order logic and a set of MKNF rules $\mathcal{P}$. We denote this translation as $\pi(\mathcal{O})$. Rules in $\mathcal{P}$ are of the form:

$\mathbf{K} a_{1}, \ldots, \mathbf{K} a_{k} \leftarrow \mathbf{K} a_{k+1}, \ldots, \mathbf{K} a_{m}, \operatorname{not} a_{m+1}, \ldots, \operatorname{not} a_{n}$

In the above, $a_{1}, \ldots, a_{n}$ are function-free first-order atoms of the form $p\left(t_{1}, \ldots, t_{i}\right)$ where $p$ is a predicate and $t_{1}, \ldots, t_{i}$ are either constants or variables, with $k \geq 1$ and $m, n, i \geq 0$. A rule $r$ in $\mathcal{P}$ is $D L$-safe if for every variable present in $r$, there is an occurence of that variable in the rule's positive body inside a predicate that does not occur in $\mathcal{K}$ 's description logic knowledge base.
A hybrid MKNF knowledge base $\mathcal{K}$ is DL-safe if every rule in $\mathcal{P}$ is DL-safe. A knowledge base that is not DLsafe may not be decidable (Motik and Rosati 2010). This constraint restricts the assignment of variables in $\mathcal{P}$ to names explictly referenced in the grounded $\mathcal{P}$. Let $\pi(\mathcal{P})$ denote rule set $\mathcal{P}$ 's corresponding MKNF formula:

$$
\begin{aligned}
\pi(\mathcal{P}) & =\bigwedge_{r \in \mathcal{P}} \pi(r), \text { where } \\
\pi(r) & =\forall \vec{x}\left(\bigvee_{i=1}^{k} \mathbf{K} a_{i} \subset \bigwedge_{i=k+1}^{m} \mathbf{K} a_{i} \wedge \bigwedge_{i=m+1}^{n} \operatorname{not} a_{i}\right)
\end{aligned}
$$

where $\vec{x}$ is the vector of free variables found in $r$.

The semantics of a hybrid MKNF knowledge base $\mathcal{K}$ is obtained by applying both transformations to $\mathcal{O}$ and $\mathcal{P}$ and placing $\mathcal{O}$ within a $\mathbf{K}$ operator, i.e. $\pi(\mathcal{K})=\pi(\mathcal{P}) \wedge \mathbf{K} \pi(\mathcal{O})$. We use $\mathcal{P}, \mathcal{O}$, and $\mathcal{K}$ in place of $\pi(\mathcal{P}), \pi(\mathcal{O})$, and $\pi(\mathcal{K})$ respectively when it is clear from context that the respective translated variant is intended. We refer to formulas of the form $\mathbf{K} a$ and not $a$, where $a$ is a first-order atoms, as $\mathbf{K}$-atoms and not-atoms respectively, and we refer to them collectively as modal-atoms. When it is clear from context, we may write a bare atom $a$ in place of a $\mathbf{K}$-atom $\mathbf{K} a$. In the rest of paper, we sometimes refer to disjunctive hybrid MKNF knowledge bases simply as knowledge bases for abbreviation, or normal knowledge bases if each rule in the knowledge base has exactly one atom in the head.

We now outline some definitions and conventions. For a hybrid MKNF knowledge base $\mathcal{K}=(\mathcal{O}, \mathcal{P})$, we denote the set of all $\mathbf{K}$-atoms found within $\mathcal{P}$ using $\mathrm{KA}(\mathcal{K})$ where

$$
\begin{aligned}
\mathrm{KA}(\mathcal{K})=\{\mathbf{K} a \mid & \text { either } \mathbf{K} a \text { or not } a \text { occurs } \\
& \text { in the head or body of a rule in } \mathcal{P}\}
\end{aligned}
$$

We use head $(r)$ to denote the set of $\mathbf{K}$-atoms in the head of the rule $r, \operatorname{body}^{-}(r)$ to denote the set of not-atoms in the body of $r$, and we use $\mathbf{K}\left(\operatorname{bod} y^{-}(r)\right)$ to denote the same set with each occurence of not replaced with $\mathbf{K}$, i.e. $\mathbf{K}\left(\operatorname{bod} y^{-}(r)\right)=\left\{\mathbf{K} a \mid \operatorname{not} a \in \operatorname{bod} y^{-}(r)\right\}$. We also use $\operatorname{body}^{+}(r)$ to denote the set of $\mathbf{K}$-atoms from the positive body of the rule $r$. The objective knowledge of a hybrid MKNF knowledge base $\mathcal{K}$ w.r.t. a set of $\mathbf{K}$-atoms $S \subseteq$ $\mathrm{KA}(\mathcal{K})$ is the set of first-order formulas $\{\pi(\mathcal{O})\} \cup\{a \mid \mathbf{K} a \in$ $S\}$. We denote this set as $\mathrm{OB}_{\mathcal{O}, S}$.

A (partial) partition of $\mathrm{KA}(\mathcal{K})$ is a nonoverlapping pair of subsets of $\mathrm{KA}(\mathcal{K})$ usually denoted as $(T, F)$. $\mathbf{K}$-atoms in $T$ are said to be true and $\mathbf{K}$-atoms in $\mathrm{F}$ are said to be false. A partition is total if $T \cup F=\mathrm{KA}(\mathcal{K})$. A dependable partition is a partial partition $(T, F)$ with the additional restriction that $\mathrm{OB}_{\mathcal{O}, T} \cup\{\neg b\}$ is consistent for each $\mathbf{K} b \in F$ or $\mathrm{OB}_{\mathcal{O}, T}$ is consistent if $F$ is empty. We add this partition variant for convenience and note that a partial partition that is not dependable may not be extended to an MKNF model. In practice, a solver will include direct consequences of $\mathrm{OB}_{\mathcal{O}, T}$ in $T$ and it will only operate on dependable partitions. We denote the partition induced by the body of a rule $r$ with $\operatorname{bod} y(r)=\left(\operatorname{bod} y^{+}(r), \mathbf{K}\left(\operatorname{bod} y^{-}(r)\right)\right)$. A rule body is applicable w.r.t. a partition $(T, F)$ if $\operatorname{body}(r) \sqsubseteq(T, F)$, i.e., if $b o d y^{+}(r) \subseteq T$ and $\mathbf{K}\left(\operatorname{bod} y^{-}(r)\right) \subseteq F$. We say that an 
MKNF interpretation $M$ of $\mathcal{K}$ induces a partition $(T, F)$ if

$$
\bigwedge_{\mathbf{K} a \in T} M \models_{M K N F} \mathbf{K} a \wedge \bigwedge_{\mathbf{K} a \in F} M \models_{M K N F} \neg \mathbf{K} a
$$

Note that the partition $\left(T^{*}, F^{*}\right)$ induced by an MKNF model $M$ is unique and dependable. For a partition $(T, F)$ that is a subset of this $\left(T^{*}, F^{*}\right)$, i.e., $(T, F) \sqsubseteq\left(T^{*}, F^{*}\right)$, we say that $(T, F)$ can be extended to an MKNF model; such a partition is also dependable. Throughout this work and without loss of generality we assume that $\mathcal{P}$ is ground, i.e., it does not contain variables.

\section{Unfounded Sets}

First defined for normal logic programs by van Gelder et al. (1991), unfounded sets encapsulate the notion that some atoms can be inferred to be false w.r.t. a partial interpretation. That is to say that given a partial partition $(T, F)$ of $\mathrm{KA}(\mathcal{K})$, an unfounded set of a knowledge base $\mathcal{K}$ w.r.t. $(T, F)$ is a set where every atom is false in every instance where $(T, F)$ can be extended to a model.

A head-cut $R \subseteq \mathcal{P} \times \mathrm{KA}(\mathcal{K})$ is a set of rule atom pairs such that a rule $r \in \mathcal{P}$ occurs in at most one pair in $R$ and for every pair $(r, h) \in R$ we have $\mathbf{K} h \in \operatorname{head}(r)$. We use head $(R)$ to denote the set $\{h \mid(r, h) \in R\}$ where $R$ is a head-cut.

Definition 2. Let $\mathcal{K}=(\mathcal{O}, \mathcal{P})$ be a disjunctive hybrid $M K N F$ knowledge base and $(T, F)$ a partial partition of $\mathrm{KA}(\mathcal{K})$. A set $X$ of $\boldsymbol{K}$-atoms is an unfounded set of $\mathcal{K}$ w.r.t. $(T, F)$ if for each $\mathbf{K}$-atom $\mathbf{K} a \in X$ and each headcut $R$ such that:

1. head $(R) \cup \pi(\mathcal{O}) \models a$ (with $\mathcal{O}, R$ can derive $\mathbf{K}$ a), and

2. head $(R) \cup O B_{\mathcal{O}, T} \cup\{\neg b\}$ is consistent for each $\mathbf{K} b \in F$ and head $(R) \cup O B_{\mathcal{O}, T}$ is consistent if $F$ is empty (the partition ( $T \cup$ head $(R), F)$ is dependable),

there is a pair $(r, h) \in R$ such that at least one of the following conditions hold:

i. body ${ }^{+}(r) \cap(F \cup X) \neq \emptyset$ (r positively depends on false or unfounded atoms),

ii. $\mathbf{K}\left(\right.$ body $\left.y^{-}(r)\right) \cap T \neq \emptyset$ ( $r$ negatively depends on true atoms), or

iii. head $(r) \cap T \neq \emptyset$ (the head of $r$ is already satisfied by T)

$\boldsymbol{K}$-atoms that are found in unfounded sets are called unfounded atoms.

We illustrate some general characteristics of this definition of unfounded sets with the following example.

Example 1. Let $\mathcal{K}=(\mathcal{O}, \mathcal{P})$ where

$$
\begin{aligned}
\mathcal{O}= & \left\{\left(a \supset a^{\prime}\right) \wedge\left(b \supset b^{\prime}\right) \wedge \neg f\right\} \\
\mathcal{P}= & \{\mathbf{K} f \leftarrow \mathbf{K} b ; \\
& \mathbf{K} a \leftarrow \mathbf{n o t} b ; \\
& \mathbf{K} a, \mathbf{K} b, \mathbf{K} c \leftarrow ; \\
& \left.\mathbf{K} a^{\prime} \leftarrow \mathbf{K} a^{\prime} ; \mathbf{K} b^{\prime} \leftarrow \mathbf{K} b^{\prime}\right\}
\end{aligned}
$$

Let $(T, F)$ be the dependable partition $(\{\mathbf{K} b\}, \emptyset)$. The $\mathbf{K}$ atom $\mathbf{K} f$ is an unfounded atom w.r.t. $(T, F)$ because $\mathbf{K} f$ creates an inconsistency in $\mathcal{O}$. $\mathbf{K} a$ is an unfounded atom because the only way of deriving $\mathbf{K}$ a relies on $\neg \mathbf{K} b$ which contradicts T. The $\mathbf{K}$-atom $\mathbf{K} a^{\prime}$ is unfounded because $\mathbf{K} a$ is unfounded and $\mathbf{K} b^{\prime}$ is not unfounded because $O B_{\mathcal{O}, T} \models b$. Lastly, $\mathbf{K} c$ is an unfounded atom because the only rule that can derive $\mathbf{K} c$ has another head-atom $(\mathbf{K} b)$ in $T$.

A head-cut $R$ that satisfies the first two conditions of the definition above is a set of rules that may be used in conjunction with $(T, F)$ and $\mathcal{O}$ to derive $\mathbf{K} a$. A $\mathbf{K}$-atom is unfounded only if every such head-cut has a pair in it that meets one of the conditions $i$ through iii. Note that if $(T, F)$ is dependable, then it is impossible to derive a $\mathbf{K}$-atom found in $F$ without violating condition 2 because an empty head-cut can be used to derive any $\mathbf{K}$-atom found in $T$. We demonstrate this property in the following example.

Example 2. Let $\mathcal{K}=(\mathcal{O}, \mathcal{P})$ where

$$
\begin{aligned}
& \mathcal{O}=\{a \supset b\}, \text { and } \\
& \mathcal{P}=\{\mathbf{K} a \leftarrow \operatorname{not} b ; \mathbf{K} b \leftarrow \operatorname{not} a\}
\end{aligned}
$$

The dependable partition $(\{\mathbf{K} b\},\{\mathbf{K} a\})$ is the only total dependable partition induced by an MKNF model of $\mathcal{K}$. Suppose we have the dependable partition $(T, F)=(\{\mathbf{K} a\}, \emptyset)$. Neither $\mathbf{K} a$ nor $\mathbf{K} b$ is an unfounded atom w.r.t. $(T, F)$ : when $R=\emptyset$ we have head $(R) \cup O B_{\mathcal{O}, T} \models a$ and $\operatorname{head}(R) \cup O B_{\mathcal{O}, T} \models b$.

Now suppose that $(T, F)=(\emptyset,\{\mathbf{K} a\}) ;$ The $\mathbf{K}$-atom $\mathbf{K} a$ is an unfounded atom w.r.t. $(T, F)$. The only head-cut that can derive $\mathbf{K} a$ is the set $R=\{(\mathbf{K} a \leftarrow \operatorname{not} b, a)\}$, however, head $(R) \cup O B_{\mathcal{O}, T} \cup\{\neg a\}$ can be rewritten as $\{a\} \cup O B_{\mathcal{O}, T} \cup$ $\{\neg a\}$ which is inconsistent.

Under Definition 2, atoms in $T$ cannot be unfounded if $(T, F)$ is dependable. Note that if $(T, F)$ is not dependable, then every set $X \subseteq \mathrm{KA}(\mathcal{K})$ is an unfounded set w.r.t. $(T, F)$. In the following, we formally establish that no $\mathbf{K}$-atom in $T$ can be an unfounded atom w.r.t. $(T, F)$.

Lemma 1 ( $T$ is disjoint from any unfounded set). Let $U$ be an unfounded set of a disjunctive knowledge base $\mathcal{K}$ w.r.t. a dependable partition $(T, F)$ of $\mathrm{KA}(\mathcal{K})$. We have $T \cap U=\emptyset$.

Proof. Assume for the sake of contradiction that $U \cap T \neq$ $\emptyset$, and let $\mathbf{K} a \in U \cap T$. Because $U$ is an unfounded set w.r.t. $(T, F)$ we have for every head-cut $R$ such that $\operatorname{head}(R) \cup \mathrm{OB}_{\mathcal{O}, T} \models a, \mathrm{OB}_{\mathcal{O}, T} \cup\{\neg b\}$ is consistent for each $\mathbf{K}$-atom $\mathbf{K} b \in F$, and $\mathrm{OB}_{\mathcal{O}, T}$ is consistent, that there is a pair $(r, h) \in R$ such that one of the conditions $i$, $i i$, or $i i i$ is satisfied. Let $R=\emptyset$. We have $\operatorname{head}(R) \cup \mathrm{OB}_{\mathcal{O}, T} \models a$ because $\mathbf{K} a \in T$. Because $(T, F)$ is dependable, $\mathrm{OB}_{\mathcal{O}, T} \cup\{\neg b\}$ is consistent for each $\mathbf{K}$-atom $\mathbf{K} b \in F$, and head $(R) \cup \mathrm{OB}_{\mathcal{O}, T}$ is consistent. However, there does not exist a pair $(r, h) \in R$ because $R$ is empty, a contradiction.

The property demonstrated in Lemma 1 is inherited from the definition of unfounded sets for normal hybrid MKNF knowledge bases (Ji, Liu, and You 2017). This is 
quite different from the definition of unfounded sets for disjunctive logic programs: Leone et al. (1997) refer to (partial) partitions (called interpretations in their context) where no atom in $T$ is unfounded (under their own definition of unfounded sets) as unfounded-free. In some respects, unfounded sets under Leone et al. (1997) can doubt the truth of $\mathbf{K}$-atoms in $T$. Since unfounded atoms are assumed to be false, an unfounded set w.r.t. $(T, F)$ that shares $\mathbf{K}$-atoms with $T$ is proof that $(T, F)$ cannot be extended to a model. As shown in Lemma 1, Definition 2 lacks this property. We illustrate this difference in the following example.

Example 3. Let $\mathcal{K}=(\emptyset, \mathcal{P})$ where $\mathcal{P}=\{\mathbf{K} a, \mathbf{K} b \leftarrow\}$ and construct the dependable partition $(T, F)=(\{\mathbf{K} a, \mathbf{K} b\}, \emptyset)$. Under Leone et al.'s definition, both $\{\mathbf{K} a\}$ and $\{\mathbf{K} b\}$ are unfounded sets w.r.t. $(T, F)$, however, the set $\{\mathbf{K} a, \mathbf{K} b\}$ is not an unfounded set w.r.t. $(T, F)$. Under Definition 2, none of the three aforementioned sets are unfounded sets w.r.t. $(T, F)$ due to Lemma 1 .

Leone et al. show that the partial partitions that have the unfounded-free property and satisfy every rule in $\mathcal{P}$ are precisely the partial partitions that can be extended to stable models (1997). In the example above, the dependable partition $(T, F)=(\{\mathbf{K} a, \mathbf{K} b\}, \emptyset)$ cannot be extended to an MKNF model and neither $\mathbf{K} a$ nor $\mathbf{K} b$ is an unfounded atom w.r.t. $(T, F)$. This indicates that unfounded sets under Definition 2 cannot be used to determine whether a partition can be extended to an MKNF model in the same way as Leone et al. We demonstrate that this is the case even for a normal knowledge base with an empty ontology.

Example 4. Let $\mathcal{K}=(\emptyset, \mathcal{P})$ where $\mathcal{P}=\{\mathbf{K} a \leftarrow \operatorname{not} a\}$. Note that $\mathcal{K}$ does not have an MKNF model. The two possible total partitions are $\left(T_{1}, F_{1}\right)=(\emptyset,\{\mathbf{K} a\})$ and $\left(T_{2}, F_{2}\right)=(\{\mathbf{K} a\}, \emptyset)$. Under both Definition 2 and Leone et al.'s definition of unfounded sets, the only unfounded set w.r.t. $\left(T_{1}, F_{1}\right)$ is $\emptyset$. Like Leone et al. , we can determine that $\left(T_{1}, F_{1}\right)$ is not an $M K N F$ model of $\mathcal{K}$ because there is a rule $r \in \mathcal{P}$ such that $\operatorname{body}(r) \sqsubseteq\left(T_{1}, F_{1}\right)$ and head $(r) \cap T_{1}=\emptyset$. Under Leone et al.'s definition, the set $\{\mathbf{K} a\}$ is an unfounded set of $\mathcal{P}$ w.r.t. $\left(T_{2}, F_{2}\right)$, however, $\{\mathbf{K} a\}$ is not an unfounded set of $\mathcal{K}$ w.r.t. $\left(T_{2}, F_{2}\right)$ under Definition 2. Critically, we cannot use Definition 2 to conclude that there is no MKNF model that induces $\left(T_{2}, F_{2}\right)$.

The above example demonstrates a limitation that prevents unfounded sets from being used as a mechanism for MKNF model checking. This limitation is also present in the unfounded sets defined by Ji et al. (2017), however, it does not inhibit unfounded sets from being useful in a solver. Following Ji et al. (2017) and Leone et al. (1997), we show that unfounded sets in Definition 2 are closed under union. First we note that condition iii of Definition 2 (head $(r) \cap T \neq \emptyset)$ does not depend on the unfounded set $X$ like it does in Leone et al.'s definition (in this context, $(\operatorname{head}(r) \backslash X) \cap T \neq \emptyset)$. The property that all dependable partitions are unfounded-free (Lemma 1) removes the need for an additional restriction on partitions as is needed for disjunctive logic programs (Leone, Rullo, and Scarcello 1997). Applying Lemma $1,(\operatorname{head}(r) \backslash X) \cap T \neq \emptyset$ can be rewritten as $\operatorname{head}(r) \cap T \neq \emptyset$. We formally demonstrate that unfounded sets by Definition 2 are closed under union and that there exists a greatest unfounded set in the following proposition.

Proposition 1 (Existence of a greatest unfounded set). Given a disjunctive hybrid MKNF knowledge base $\mathcal{K}=$ $(\mathcal{O}, \mathcal{P})$ and a partial partition $(T, F)$ of $\mathrm{KA}(\mathcal{K})$, there exists a greatest unfounded set $U_{\mathcal{K}}(T, F)$ such that for every unfounded set $X$ of $\mathcal{K}$ w.r.t. $(T, F)$ we have $U_{\mathcal{K}}(T, F) \supseteq X$.

Proof. We show that unfounded sets are closed under union and the existence of a greatest unfounded set directly follows. Let $X_{a}$ and $X_{b}$ be unfounded sets of $\mathcal{K}$ w.r.t. a partial partition $(T, F)$ of $\mathrm{KA}(\mathcal{K})$. We show that the set $X_{c}=$ $X_{a} \cup X_{b}$ is an unfounded set of $\mathcal{K}$ w.r.t. $(T, F)$. If $(T, F)$ is not dependable, then every set $X \subseteq \mathrm{KA}(\mathcal{K})$ is an unfounded set of $\mathcal{K}$ w.r.t. $(T, F)$ including $X_{c}$. Assume that $(T, F)$ is dependable and for the sake of contradiction, assume $X_{c}$ is not an unfounded set. For some $\mathbf{K}$-atom $\mathbf{K} a \in X_{c}$ we have a head-cut $R$ s.t. conditions $1\left(\operatorname{head}(R) \cup \mathrm{OB}_{\mathcal{O}, T} \models a\right)$ and 2 (head $(R) \cup \mathrm{OB}_{\mathcal{O}, T} \cup\{\neg b\}$ is consistent for each $\mathbf{K} b \in F$ or head $(R) \cup \mathrm{OB}_{\mathcal{O}, T}$ is consistent if $F$ is empty) hold. In this head-cut, there is a pair $(r, a)$ such that none of the conditions $i\left(b o d y^{+}(r) \cap\left(X_{c} \cup F\right) \neq \emptyset\right)$, $i i\left(b o d y^{-}(r) \cap T \neq \emptyset\right)$, or iii (head $(r) \cap T \neq \emptyset$ ) hold. For simplicity, assume K $a \in X_{a}$ (proof is identical if $\mathbf{K} a \in X_{b}$ ). If $b o d y^{+}(r) \cap\left(X_{a} \cup F\right) \neq \emptyset$ then we have $b o d y^{+}(r) \cap\left(X_{c} \cup F\right) \neq \emptyset$ and it follows that $X_{c}$ is an unfounded set.

This property is a natural result of Lemma 1 and differs from Leone et al.'s unfounded sets, which are closed under union only if $(T, F)$ is unfounded-free.

A solver can use any unfounded set to extend a dependable partition's false atoms without altering which models it finds. We now relate unfounded sets to MKNF models.

Proposition 2. Let $\left(T^{*}, F^{*}\right)$ be the partition induced by an MKNF model of a disjunctive hybrid MKNF knowledge base $\mathcal{K}$. For any dependable partition $(T, F) \sqsubseteq\left(T^{*}, F^{*}\right)$, $U_{\mathcal{K}}(T, F) \cap T^{*}=\emptyset$.

Proof. Let $M$ be the MKNF model that induces $\left(T^{*}, F^{*}\right)$. Note that $\left(T^{*}, F^{*}\right)$ is total and dependable. Let $(T, F) \sqsubseteq$ $\left(T^{*}, F^{*}\right)$ and $U$ be an unfounded set of $\mathcal{K}$ w.r.t. $(T, F)$. We show that $U \cap T^{*}=\emptyset$ and it follows that $U_{\mathcal{K}}(T, F) \cap T^{*}=\emptyset$. Assume for the sake of contradiction that $U \cap T^{*} \neq \emptyset$. With $B=U \cap T^{*}$, let us construct an MKNF interpretation $M^{\prime}$ such that

$M^{\prime}=\left\{I \mid I \models \mathrm{OB}_{\mathcal{O}, T}\right.$ and $I \models t$ for each $\left.\left.\mathbf{K} t \in T^{*} \backslash B\right\}\right\}$

The dependable partition induced by $M^{\prime}$ is $\left(T^{*} \backslash B, F^{*} \cup\right.$ $B)$. For each $b \in B, \mathrm{OB}_{\mathcal{O}, T} \not=b$, thus $M^{\prime} \supset$ $M$. We will derive a contradiction by showing $U$ is not an unfounded set of $\mathcal{K}$ w.r.t. $(T, F)$. By construction, $\left.\left(I, M^{\prime}, M\right)\right|_{M K N F} \pi(\mathcal{O})$ for each $I \in M^{\prime}$. We now show $\forall I \in M^{\prime},\left(I, M^{\prime}, M\right) \models{ }_{M K N F} \pi(\mathcal{P})$, and it then follows that $M$ is not an MKNF model which leads to a contradiction. Let $\left(T^{*} \backslash B, F\right)$ be the induced partition (from $M^{\prime}$ for true $\mathbf{K}$-atoms in $T^{*} \backslash B$ and from $M$ for false $\mathbf{K}$-atoms in $F$ ). Observe that if a rule $r \in \mathcal{P}$ is not satisfied w.r.t. $\left(T^{*} \backslash B, F\right)$ then it must be the case that $\operatorname{body}(r) \sqsubseteq\left(T^{*} \backslash B, F\right)$, 
$\operatorname{head}(r) \cap T^{*} \neq \emptyset$, and $\operatorname{head}(r) \cap\left(T^{*} \backslash B\right)=\emptyset$. That is, $r$ is a rule whose body is satisfied by $\left(T^{*} \backslash B, F\right)$ but all true atoms in its head come from $B$, because $M$ satisfies all rules in $\mathcal{P}$.

Let $R=\{(r, h)\}$ and $b$ be some atom from head $(r) \cap B$. Conditions 1 and 2 of Definition 2 are met for $R$ to test if $U$ is an unfounded set of $\mathcal{K}$ w.r.t. $(T, F)$. We show that none of the conditions $i$ through $i i i$ are met by $R$, as such $U$ cannot be an unfounded set w.r.t. $(T, F)$, which leads to a contradiction. First, body $y^{+}(r) \subseteq T^{*} \backslash B$ gives us $\operatorname{body}^{+}(r) \cap(F \cup U)=\emptyset$ (which violates condition $i$ ). Then, from $\mathbf{K}\left(b o d y^{-}(r)\right) \subseteq F$, we derive $\mathbf{K}\left(b o d y^{-}(r)\right) \cap T=\emptyset$ (which violates condition $i$ ). Finally, using head $(r) \cap T^{*} \subseteq$ $B$ and $B \cap T=\emptyset$ (Lemma 1), we obtain head $(r) \cap T=\emptyset$ (which falsifies condition $i$ ii). We have shown $U \cap T^{*}=\emptyset$, as desired.

We have shown that if a dependable partition $(T, F)$ can be extended to an MKNF model, no unfounded set of $\mathcal{K}$ w.r.t. $(T, F)$ may overlap with the true atoms in the model. It follows directly from Proposition 2 that the following analogous property holds for unfounded atoms w.r.t. $(T, F)$.

Corollary 2. Let $\left(T^{*}, F^{*}\right)$ be the partition induced by an MKNF model $M$ of a disjunctive hybrid MKNF knowledge base $\mathcal{K}$. Then, for any dependable partition $(T, F) \sqsubseteq$ $\left(T^{*}, F^{*}\right), M \models M K N F \neg \mathbf{K} u$ for all $u \in U_{\mathcal{K}}(T, F)$.

With these properties, we have shown that unfounded sets can be used to extend a partition without missing any models, i.e., if $(T, F)$ can be extended to an MKNF model $M$ then $(T, F \cup U)$ can be extended to the same model $M$ for any unfounded set $U$ w.r.t. $(T, F)$.

\section{Computing Unfounded Sets}

Due to the inconsistencies that can arise in connection with $\mathcal{O}$, computing the greatest unfounded set w.r.t. a partial partition is intractable in general.

Example 5. Let $\mathcal{K}=(\mathcal{O}, \mathcal{P})$ where $\mathcal{O}=\neg(a \wedge b)$ and

$$
\mathcal{P}=\{\mathbf{K} a \leftarrow \operatorname{not} b ; \mathbf{K} b \leftarrow \operatorname{not} a ; \mathbf{K} c \leftarrow \mathbf{K} c\}
$$

Under Definition 2, $\mathbf{K} c$ is an unfounded atom w.r.t. $(\emptyset, \emptyset)$, however, with the $V_{\mathcal{K}}^{(\emptyset, \emptyset)}$ operator defined by Ji et al. (2017) we have $\boldsymbol{l f} \boldsymbol{p}\left(V_{\mathcal{K}}^{(\emptyset, \emptyset)}\right)=\mathrm{KA}(\mathcal{K})$ which misses $\mathbf{K} c$ as an unfounded atom. ${ }^{1}$ It's clear that a similar operator for disjunctive knowledge bases would have the same limitation.

In the following, we first give a formal proof of intractability and then we construct an operator for hybrid MKNF knowledge bases with disjunctive rules that adopts the same approximation technique used by $\mathrm{Ji}$ et al. in their $V_{\mathcal{K}}^{(T, F)}$ operator (2017) for hybrid MKNF knowledge bases with normal rules.

We now show that deciding whether an atom of a normal hybrid MKNF knowledge base is unfounded is coNP-hard

\footnotetext{
${ }^{1}$ This is because in the least fixed point computations of the $V_{\mathcal{K}}^{(T, F)}$ operator, a default negation not $q$ is true if $\mathbf{K} q$ is not known to be true, and as such, both $\mathbf{K} a$ and $\mathbf{K} b$ are derived in the first iteration which leads to inconsistency with $\mathcal{O}$.
}

by comparing the head-cuts that need to be considered to determine unfoundedness with the SAT assignments that need to be considered to determine the satisfiability of a 3SAT problem.

Proposition 3. Let $\mathcal{K}=(\mathcal{O}, \mathcal{P})$ be a normal hybrid MKNF knowledge base such that the entailment relation $O B_{\mathcal{O}, S}=$ a can be checked in polynomial time for any set $S \subseteq \mathrm{KA}(\mathcal{K})$ and for any $\boldsymbol{K}$-atom $\mathbf{K} a \in \mathrm{KA}(\mathcal{K})$. Determining whether a $\mathbf{K}$-atom $\mathbf{K} a \in \mathrm{KA}(\mathcal{K})$ is an unfounded atom of $\mathcal{K}$ w.r.t. a dependable partition $(T, F)$ of $\mathrm{KA}(\mathcal{K})$ is coNP-hard.

Proof. We show that the described problem is coNP-hard. The 3SAT problem is well known to be NP-complete (Sipser 1996). Let $S A T$ be an instance of 3 SAT in conjunctive normal form such that $C L A U S E=\left\{c_{1}, c_{2}, \ldots, c_{n}\right\}$ is the set of clauses in $S A T$ and $V A R=\left\{v_{1}, v_{2}, \ldots, v_{n}\right\}$ is the set of variables in $S A T$. Determining whether $S A T$ is unsatisfiable is coNP-hard. We construct a normal hybrid MKNF knowledge base $\mathcal{K}=(\mathcal{O}, \mathcal{P})$ s.t.

$\mathcal{O}=\left\{v_{i}^{u} \oplus v_{i}^{f} \oplus v_{i}^{t} \mid\right.$ for each $v_{i} \in V A R$

where $\oplus$ is exclusive-or $\} \cup$

$$
\begin{aligned}
& \left\{\left(\bigwedge_{v_{i} \in V A R} \neg v_{i}^{u} \Longleftrightarrow \text { total }\right), \text { total } \supset \text { sat }\right\} \cup \\
& \left\{\text { clause }_{i} \vee \neg \text { total } \mid \text { for each clause } c_{i} \in C L A U S E\right. \\
& \text { where } \text { clause }_{i} \text { is a formula } \\
& \text { obtained by replacing all occurences }
\end{aligned}
$$

of $v_{i}$ and $\neg v_{i}$ in $c_{i}$ with $v_{i}^{t}$ and $v_{i}^{f}$ respectively $\}$

$\mathcal{P}=\{\mathbf{K}$ sat $\leftarrow \mathbf{K}$ sat $\} \cup$

$$
\bigcup\left\{\left\{\left(\mathbf{K} v_{i}^{t} \leftarrow \mathbf{n o t} v_{i}^{f}\right),\left(\mathbf{K} v_{i}^{f} \leftarrow \mathbf{n o t} v_{i}^{t}\right)\right\} \mid v_{i} \in V A R\right\}
$$

Note that the rule $\mathbf{K} s a t \leftarrow \mathbf{K}$ sat is only required to ensure that $\mathbf{K}$ sat is in $\mathrm{KA}(\mathcal{K})$. The time to construct the above knowledge base is linear in the number of clauses and variables in $S A T$. The first set of formulas in $\mathcal{O}$ requires exactly one of $v_{i}^{u}, v_{i}^{f}$, or $v_{i}^{t}$ to be true. This constraint is analogous to a three-valued assignment for $S A T$ where a variable $v_{i} \in V A R$ is unassigned if $v_{i}^{u}$ is true, assigned false if $v_{i}^{f}$ is true, and assigned true if $v_{i}^{t}$ is true. The second set in $\mathcal{O}$ ensures that the atom total is true if and only if no variable is unassigned. Finally, the third set of formulas ensure that $\pi(\mathcal{O})$ is inconsistent if the assignment is total and a clause in $S A T$ is not satisfied. We show that (A) For any $\mathbf{K}$-atom $\mathbf{K} a$ and set of $\mathbf{K}$-atoms $S$, the entailment relation $\mathrm{OB}_{\mathcal{O}, S}=a$ is computable in polynomial time and (B) that $\mathbf{K}$ sat is an unfounded atom of $\mathcal{K}$ w.r.t. $(\emptyset, \emptyset)$ if and only if $S A T$ is unsatisfiable.

(A) We call a set of $\mathbf{K}$-atoms $S$ total if it contains either $\mathbf{K} v_{i}^{t}$ or $\mathbf{K} v_{i}^{f}$ for each variable $v_{i} \in V A R$. Note that for a variable $v_{i} \in V A R$, the set $\mathrm{KA}(\mathcal{K})$ only contains $\mathbf{K} v_{i}^{t}$ and $\mathbf{K} v_{i}^{f}$; It does not contain $\mathbf{K} v_{i}^{u}$. Let $S \subseteq \mathrm{KA}(\mathcal{K})$. We show that we can, in polynomial time, determine whether $S \cup \pi(\mathcal{O})$ is consistent. We split cases where $S$ is total and 
where it is not. First, assume $S$ is not total: For some variable $v_{i} \in V A R$, neither $\mathbf{K} v_{i}^{t}$ nor $\mathbf{K} v_{i}^{f}$ is in $S$. By fixing $v_{i}^{u}$ to be true in a consistent first-order interpretation $I$ of $S \cup \pi(\mathcal{O})$, we ensure the atom total is false. If the atom total is false, we can determine whether $\mathrm{OB}_{\mathcal{O}, S}$ is consistent in polynomial time because we only need to consider the first two sets of formulas in $\mathcal{O}$. If $S$ is total, we can, in polynomial time, verify that $S \cup \pi(\mathcal{O})$ is consistent by checking that only one of $v_{i}^{t}$ or $v_{i}^{f}$ is present in $S$ and that every clause clause $_{i}$ is satisfied. After determining whether $S \cup \pi(\mathcal{O})$ is consistent, we can quickly check the relations $\mathrm{OB}_{\mathcal{O}, S} \models v_{i}^{t}$ and $\mathrm{OB}_{\mathcal{O}, S} \models v_{i}^{f}$ for any variable $v_{i} \in V A R$ : Assuming $S \cup \pi(\mathcal{O})$ is consistent, the entailment relation $\mathrm{OB}_{\mathcal{O}, S} \models v_{i}^{t}$ (resp. $\mathrm{OB}_{\mathcal{O}, S}=v_{i}^{f}$ ) holds if and only if $\mathbf{K} v_{i}^{t} \in S$ (resp. $\mathbf{K} v_{i}^{f} \in S$ ). When $S \cup \pi(\mathcal{O})$ is consistent, the entailment relation $\mathrm{OB}_{\mathcal{O}, S} \models$ total holds if and only if $S$ is total. Finally, we have $\mathrm{OB}_{\mathcal{O}, S} \models$ sat if and only if $\mathbf{K}$ sat $\in S$ or $\mathrm{OB}_{\mathcal{O}, S}=$ total. If $S \cup \pi(\mathcal{O})$ is inconsistent, the entailment relation $\mathrm{OB}_{\mathcal{O}, S} \models \mathbf{K} a$ holds vacuously for any $\mathbf{K} a \in \mathrm{KA}(\mathcal{K})$.

(B) When determining whether the $\mathbf{K}$-atom $\mathbf{K}$ sat is unfounded w.r.t. $(\emptyset, \emptyset)$, we must consider each way to select a head-cut $R$. We show that there is a correspondence between the head-cuts that can disprove the unfoundedness of $\mathbf{K}$ sat w.r.t. $(\emptyset, \emptyset)$ and total sat assignments for $S A T$. Let $X=$ $\{\mathbf{K}$ sat $\}$ be a set that is possibly unfounded w.r.t. $(\emptyset, \emptyset)$. Observe that a larger unfounded set $X^{\prime} \supset X$ w.r.t. $(\emptyset, \emptyset)$ cannot exist unless $X$ is an unfounded set w.r.t. $(\emptyset, \emptyset)$. A head-cut $R$ cannot be used to disprove the unfoundedness of $\mathbf{K}$ sat if either condition 1 or 2 of Definition 2 do not hold. Before creating a mapping between head-cuts and sat assignments for $S A T$, we exclude head-cuts that cannot be used to disprove the unfoundedness of $\mathbf{K}$ sat, i.e., conditions 1 and 2 of Definition 2 are met and $i$, ii, and iii do not hold. Firstly, we exclude head-cuts that contain the pair $(r, s a t)$ because $b o d y^{+}(r) \cap X \neq \emptyset$. We further exclude any headcut $R$ containing a pair of pairs $\left(r_{0}, v_{i}^{t}\right)$ and $\left(r_{1}, v_{i}^{f}\right)^{2}$ because $\operatorname{head}(R) \cup \mathrm{OB}_{\mathcal{O}, \emptyset}$ is inconsistent. Thirdly, we exclude any head-cuts that do not contain either $\left(r_{0}, v_{i}^{t}\right)$ or $\left(r_{1}, v_{i}^{f}\right)$ for each variable $v_{i} \in V A R$ noting that if such a head-cut $R$ also meets the previous two conditions we have $\operatorname{head}(R) \cup \mathrm{OB}_{\mathcal{O}, \emptyset} \not \models \operatorname{sat}$ (See part (A) of this proof for details). The remaining head-cuts have a one to one correspondence with total assignments for $S A T$ : if a head-cut contains a pair with $v_{i}^{t}$ (resp. $v_{i}^{f}$ ) the corresponding assignment for $S A T$ assigns $v_{i}$ to be true (resp. false). We have for every such head-cut $R$ that $h e a d(R) \cup \mathrm{OB}_{\mathcal{O}, \emptyset}=$ sat and that for every pair in $(r, h) \in R$ we have head $(r) \cap T=\emptyset$, $\operatorname{body}^{+}(r) \cap(F \cup X)=\emptyset$, and $\operatorname{body}^{-}(r) \cap T=\emptyset$. If $\operatorname{head}(R) \cup \mathrm{OB}_{\mathcal{O}, \emptyset}$ is consistent, then every clause is satisfied by the corresponding sat assignment, otherwise, the inconsistency is caused by an unsatisfied clause $\neg$ clause $_{i}$, thus the assignment does not satisfy $S A T$. If no such headcut $R$ exists such that head $(R) \cup \mathrm{OB}_{\mathcal{O}, \emptyset}$ is consistent, then

\footnotetext{
${ }^{2}$ Due to the uniqueness of the second component in such a pair, there should be no confusion about which rule the first component refers to.
}

$\mathbf{K}$ sat is unfounded w.r.t. $(\emptyset, \emptyset)$ and $S A T$ is unsatisfiable. Conversely, if $S A T$ is unsatisfiable, a head-cut $R$ such that $\operatorname{head}(R) \cup \mathrm{OB}_{\mathcal{O}, \emptyset}$ is consistent and $\operatorname{head}(R) \cup \mathrm{OB}_{\mathcal{O}, \emptyset} \models$ sat does not exist, thus $\{\mathbf{K}$ sat $\}$ is an unfounded set w.r.t. $(\emptyset, \emptyset)$. We have shown that deciding whether an $\mathbf{K}$-atom is unfounded is coNP-hard.

It follows that computing the greatest unfounded set of a disjunctive hybrid MKNF knowledge base is coNP-hard. Since we are unlikely to find a way to compute $U_{\mathcal{K}}(T, F)$ in polynomial time, we are motivated to construct a polynomial operator that computes an approximation (a subset) of the greatest unfounded set. We define a family of operators $Z_{\mathcal{K}}^{(T, F)}$ where each operator induced by a dependable partition $(T, F)$ computes an approximation of the greatest unfounded set of $\mathcal{K}$ w.r.t. $(T, F)$

$$
\begin{aligned}
Z_{\mathcal{K}}^{(T, F)} & (X)= \\
& \left\{\mathbf{K} a \mid \mathrm{OB}_{\mathcal{O}, X}=a \text { for each } \mathbf{K} a \in \mathrm{KA}(\mathcal{K})\right\} \cup \\
& \{\mathbf{K} a \mid \exists r \in \mathcal{P} \text { with } \mathbf{K} a \in \operatorname{head}(r) \text { s.t. } \\
& \text { body }^{+}(r) \subseteq X \wedge \operatorname{bod} y^{+}(r) \cap F=\emptyset \wedge \\
& \mathbf{K}(b o d y-(r)) \cap T=\emptyset \wedge \text { head }(r) \cap T=\emptyset \wedge \\
& \left.\{a, \neg b\} \cup \mathrm{OB}_{\mathcal{O}, T} \text { is consistent for each } \mathbf{K} b \in F\right\}
\end{aligned}
$$

This operator is the direct result of combining the $V_{\mathcal{K}}^{(T, F)}$ operator for normal hybrid MKNF knowledge bases ( $\mathrm{Ji}, \mathrm{Liu}$, and You 2017) with the $\Phi$ operator for disjunctive logic programs (Leone, Rullo, and Scarcello 1997). It is easy to see that the $Z_{\mathcal{K}}^{(T, F)}$ operator is monotonic, and let us use $\operatorname{Atmost}_{\mathcal{K}}(T, F)$ to denote its least fixed point. This operator computes a subset of $\mathrm{KA}(\mathcal{K}) \backslash U_{\mathcal{K}}(T, F)$. In particular, if $\operatorname{Atmost}_{\mathcal{K}}(T, F) \cup \pi(\mathcal{O})$ is inconsistent, we have $\mathrm{KA}(\mathcal{K}) \backslash \operatorname{Atmost}_{\mathcal{K}}(T, F)=\emptyset$, a compromise to keep the operator computable in polynomial time.

To determine whether an atom is unfounded when there are disjunctive rules, we must consider an exponential number of head-cuts. The $Z_{\mathcal{K}}^{(T, F)}$ operator instead considers the heads of rules all at once and this can result in $\operatorname{Atmost}_{\mathcal{K}}(T, F)$ missing some unfounded atoms even if $\operatorname{Atmost}_{\mathcal{K}}(T, F) \cup \pi(\mathcal{O})$ is consistent.

Example 6. Let $\mathcal{K}=(\mathcal{O}, \mathcal{P})$ be a disjunctive hybrid MKNF knowledge base where $\mathcal{P}=\{\mathbf{K} a, \mathbf{K} b \leftarrow ; \mathbf{K} c \leftarrow$ $\mathbf{K} c\}$ and $\mathcal{O}=(a \wedge b) \supset c$. We have that $\{\mathbf{K} c\}$ is an unfounded set of $\mathcal{K}$ w.r.t. $(\emptyset, \emptyset)$. However, Atmost $\operatorname{K}_{\mathcal{K}}(T, F)$ is $\{a, b, c\}$ and $\operatorname{KA}(\mathcal{K}) \backslash\{a, b, c\} \neq U_{\mathcal{K}}(\emptyset, \emptyset)$.

We intend to identify the class of knowledge bases for which the $Z_{\mathcal{K}}^{(T, F)}$ operator does not miss unfounded atoms as a result of disjunctive heads. First we define a weak head-cut to be a set of rule atom pairs $R^{w}$ such that $R^{w} \subseteq$ $\mathcal{P} \times \mathrm{KA}(\mathcal{K})$ and $h \in \operatorname{head}(r)$ for each pair $(r, h) \in R$. Note that this definition is identical to the definition of headcuts without the constraint that a rule can appear in at most one pair in $R^{w}$; within a weak head-cut, there may be two pairs $\left(r, h_{0}\right)$ and $\left(r, h_{1}\right)$ such that $h_{0} \neq h_{1}$. In the following, we define a property that captures a subset of knowl- 
edge bases where $\operatorname{Atmost}_{\mathcal{K}}(T, F)$ computes $U_{\mathcal{K}}(T, F)$ if $\operatorname{Atmost}_{\mathcal{K}}(T, F) \cup \pi(\mathcal{O})$ is consistent.

Definition 3. A hybrid MKNF knowledge base $\mathcal{K}=(\mathcal{O}, \mathcal{P})$ is head-independent w.r.t. a dependable partition $(T, F)$ if for every $\mathbf{K}$-atom $\mathbf{K} a \in \mathrm{KA}(\mathcal{K})$ and every weak head-cut $R^{w}$ such that head $(R) \cup O B_{\mathcal{O}, T}=a$, there exists a headcut $R$ such that $R \subseteq R^{w}$ and head $(R) \cup O B_{\mathcal{O}, T} \models a$.

Head-independence means that we cannot derive atoms that we would not be able to derive using only a single atom from each rule head by using multiple atoms in the head of a rule in conjunction with the ontology. The head-independence property is violated by the knowledge base in Example 6 and it ensures that $\operatorname{Atmost}_{\mathcal{K}}(T, F) \neq$ $U_{\mathcal{K}}(T, F)$. Were we to alter the knowledge base in Example 6 such that the rule $\mathbf{K} a, \mathbf{K} b \leftarrow$ were changed to the pair of rules $\mathbf{K} a \leftarrow \operatorname{not} b$ and $\mathbf{K} b \leftarrow \operatorname{not} a$ then $\mathcal{K}$ would have head-independence. We show formally that for a head-independent knowledge base $\mathcal{K}$, the $Z_{\mathcal{K}}^{(T, F)}$ operator computes the greatest unfounded set w.r.t. $(T, F)$ if $\operatorname{Atmost}_{\mathcal{K}}(T, F) \cup \pi(\mathcal{O})$ is consistent.

Proposition 4. If $\mathcal{K}$ is head-independent w.r.t. a dependable partition $(T, F)$ and $\operatorname{Atmost}_{\mathcal{K}}(T, F) \cup \pi(\mathcal{O})$ is consistent, then $U_{\mathcal{K}}(T, F)=\mathrm{KA}(\mathcal{K}) \backslash$ Atmost $_{\mathcal{K}}(T, F)$.

Proof. First we show (1) that no K-atom computed by $\operatorname{Atmost}_{\mathcal{K}}(T, F)$ is unfounded w.r.t. $(T, F)$ and then we show (2) that every atom that is not unfounded w.r.t. $(T, F)$ is computed by $\operatorname{Atmost}_{\mathcal{K}}(T, F)$.

(1) We first show no $\mathbf{K}$-atom in $Z_{\mathcal{K}}^{(T, F)}(\emptyset)$ is unfounded. Let $\mathbf{K} a \in Z_{\mathcal{K}}^{(T, F)}(\emptyset)$. Construct a weak head-cut $R^{w}$ that contains a pair $(r, h)$ for each head $\mathbf{K}$-atom $\mathbf{K} h \in \operatorname{head}(r)$ and rule $r \in \mathcal{P}$ where $b o d y^{+}(r) \subseteq \emptyset, \mathbf{K}\left(\operatorname{bod} y^{-}(r)\right) \cap T=\emptyset$, and $\operatorname{head}(r) \cap T=\emptyset$. The weak head-cut $R^{w}$ contains every rule that was applied in the computation of $Z_{\mathcal{K}}^{(T, F)}(\emptyset)$. We have head $\left(R^{w}\right) \cup \mathrm{OB}_{\mathcal{O}, T} \models a$. Applying the headindependence condition, we obtain a head-cut $R$ such that $R \subseteq R^{w}$ and head $(R) \cup \mathrm{OB}_{\mathcal{O}, T} \models a$. For every pair $(r, h) \in R, \operatorname{body}^{+}(r) \subseteq T, \mathbf{K}\left(\operatorname{body} y^{-}(r)\right) \cap T=\emptyset$, and $\operatorname{head}(r) \cap T$. The head-cut $R$ shows that $\mathbf{K} a$ is not an unfounded atom w.r.t. $(T, F)$, thus it is not a member of any unfounded set. We show that no atom computed by a successive application of $Z_{\mathcal{K}}^{(T, F)}$, e.g., $Z_{\mathcal{K}}^{(T, F)}\left(Z_{\mathcal{K}}^{(T, F)}(\emptyset)\right)$, is unfounded w.r.t. $(T, F)$. Let $Z_{i}$ be result of applying the $Z_{\mathcal{K}}^{(T, F)}$ operator $i$ times where $Z_{0}=\emptyset$. We assume that no atom in $Z_{i}$ is unfounded w.r.t. $(T, F)$ and show the same for $Z_{i+1}$. Construct a weak head-cut $R^{w}$ that contains a pair $(r, h)$ for each head a $\mathbf{K}$-atom $\mathbf{K} h \in \operatorname{head}(r)$ and rule $r \in \mathcal{P}$ where $\operatorname{body}^{+}(r) \subseteq Z_{i}$, body $y^{-}(r) \cap T=\emptyset$, and head $(r) \cap T=\emptyset$. Let $\mathbf{K} a \in Z_{\mathcal{K}}^{(T, F)}\left(Z_{i}\right)$. We have head $\left(R^{w}\right) \cup \mathrm{OB}_{\mathcal{O}, T} \models a$. Applying the head-independence condition, we obtain a head-cut $R$ such that $R \subseteq R^{w}$ and $\operatorname{head}(R) \cup \mathrm{OB}_{\mathcal{O}, T} \models a$. Now we have for each pair $(r, h) \in R, \operatorname{body} y^{+}(r) \subseteq Z_{i}$. Knowing that no $\mathbf{K}$-atom in $Z_{i}$ is a member of an unfounded set, we conclude that $a$ is not an unfounded atom w.r.t. $(T, F)$.

(2) We show that if a $\mathbf{K}$-atom $\mathbf{K} a$ is not computed by $\operatorname{Atmost}_{\mathcal{K}}(T, F)$ and it is not an unfounded atom w.r.t. $(T, F)$, we can derive a contradiction. Let $U=$ $\mathrm{KA}(\mathcal{K}) \backslash \operatorname{Atmost}_{\mathcal{K}}(T, F)$. Let $\mathbf{K} a \in U$ be an $\mathbf{K}$-atom such that there exists a head-cut $R$ where $h e a d(R) \cup \mathrm{OB}_{\mathcal{O}, T} \models a$, $\operatorname{head}(R) \cup \mathrm{OB}_{\mathcal{O}, T}$ is consistent and head $(R) \cup \mathrm{OB}_{\mathcal{O}, T} \cup$ $\{\neg b\}$ is consistent for each $\mathbf{K} b \in F$ and for each pair $(r, h) \in R$, head $(r) \cap T=\emptyset$ and body ${ }^{-}(r) \cap T=\emptyset$. If for each pair $(r, h) \in R$ we have body ${ }^{+}(r) \not \operatorname{Atmost}_{\mathcal{K}}(T, F)$ then $U$ is an unfounded set w.r.t. $(T, F)$, otherwise $\mathbf{K} a \in$ $\operatorname{Atmost}_{\mathcal{K}}(T, F)$. Both cases contradict the initial assumptions.

For normal knowledge bases, i.e., where each rule contains only a single head-atom, the head-independence condition is satisfied automatically. If a knowledge base $\mathcal{K}$ is not head-independent, the $Z_{\mathcal{K}}^{(T, F)}$ operator computes a subset of $U_{\mathcal{K}}(T, F)$. Therefore, for a normal knowledge base and dependable partition $(T, F)$ s.t. $\operatorname{Atmost}_{\mathcal{K}}(T, F) \cup \pi(\mathcal{O})$ is consistent, we have $U_{\mathcal{K}}(T, F)=\mathrm{KA}(\mathcal{K}) \backslash \operatorname{Atmost}_{\mathcal{K}}(T, F)$. The following corollary follows directly from Proposition 4.

Corollary 3. If a knowledge base $\mathcal{K}$ is head-independent w.r.t. a dependable partition $(T, F)$ and $\operatorname{Atmost}_{\mathcal{K}}(T, F) \cup$ $\pi(\mathcal{O})$ is consistent, then the greatest unfounded set of $\mathcal{K}$ w.r.t. $(T, F)$ is computable in polynomial time.

We have shown that computing the greatest unfounded set of a normal knowledge base is coNP-hard (Proposition 3). Because $\operatorname{Atmost}_{\mathcal{K}}(T, F)$ can be computed in polynomial time, we conclude that the greatest unfounded set of a normal knowledge base $\mathcal{K}$ can be computed in polynomial time if $\operatorname{Atmost}_{\mathcal{K}}(T, F) \cup \pi(\mathcal{O})$ is consistent and the greatest unfounded set of a disjunctive knowledge base $\mathcal{K}$ can be computed in polynomial time if $\operatorname{Atmost}_{\mathcal{K}}(T, F) \cup \pi(\mathcal{O})$ is consistent and $\mathcal{K}$ is head-independent. Observe that for the knowledge base constructed in our proof of Proposition 3, $\operatorname{Atmost}_{\mathcal{K}}(T, F) \cup \pi(\mathcal{O})$ is inconsistent. We formally demonstrate the intractability of computing $U_{\mathcal{K}}(T, F)$ for a disjunctive knowledge base when $\operatorname{Atmost}_{\mathcal{K}}(T, F) \cup \pi(\mathcal{O})$ is consistent but the head-independence condition is not met.

Proposition 5. Let $\mathcal{K}=(\mathcal{O}, \mathcal{P})$ be a disjunctive hybrid $M K N F$ knowledge base such that the entailment relation $O B_{\mathcal{O}, S}=$ a can be checked in polynomial time for any set $S \subseteq \mathrm{KA}(\mathcal{K})$ and for any $\mathbf{K}$-atom $\mathbf{K} a \in \mathrm{KA}(\mathcal{K})$. Let $(T, \bar{F})$ be a dependable partition of $\mathrm{KA}(\mathcal{K})$ such that Atmost $_{\mathcal{K}}(T, F) \cup \pi(\mathcal{O})$ is consistent. Determining whether a $\mathbf{K}$-atom $\mathbf{K} a \in \mathrm{KA}(\mathcal{K})$ is an unfounded atom of $\mathcal{K}$ w.r.t. $(T, F)$ is coNP-hard.

Proof. Let $S A T$ be an instance of 3SAT in conjunctive normal form such that $C L A U S E=\left\{c_{1}, c_{2}, \ldots, c_{n}\right\}$ is the set of clauses in $S A T$, and $V A R=\left\{v_{1}, v_{2}, \ldots, v_{n}\right\}$ is the set of variables in $S A T$. In Figure 1, we construct a disjunctive hybrid MKNF knowledge base $\mathcal{K}=(\mathcal{O}, \mathcal{P})$.

Let $(T, F)=(\emptyset, \emptyset)$ and observe that $\operatorname{Atmost}_{\mathcal{K}}(T, F) \cup$ $\pi(\mathcal{O})$ is consistent $\left(\operatorname{Atmost}_{\mathcal{K}}(T, F)=\mathrm{KA}(\mathcal{K}) \backslash\{\mathbf{K}\right.$ sat $\left.\}\right)$. We show that (A) For any $\mathbf{K}$-atom $\mathbf{K} a$ and set of $\mathbf{K}$-atoms $S$, the entailment relation $\mathrm{OB}_{\mathcal{O}, S} \models a$ is computable in polynomial time and (B) that $\mathbf{K}$ sat is an unfounded atom of $\mathcal{K}$ w.r.t. $(\emptyset, \emptyset)$ if and only if $S A T$ is unsatisfiable.

(A) Observe that $\mathrm{KA}(\mathcal{K}) \cup \pi(\mathcal{O})$ is consistent, therefore, $S \cup \pi(\mathcal{O})$ is consistent for any set of $\mathbf{K}$-atoms $S \subseteq \mathrm{KA}(\mathcal{K})$. 


$$
\begin{aligned}
\mathcal{O}= & \left\{\left(v_{i}^{f} \vee v_{i}^{t}\right) \oplus v_{i}^{u} \mid \text { for each } v_{i} \in V A R\right. \\
& \text { where } \oplus \text { is exclusive-or }\} \cup \\
& \left\{\left(v_{i}^{f} \wedge v_{i}^{t}\right) \supset \text { sat } \mid \text { for each } v_{i} \in V A R\right\} \cup \\
& \left\{\left(\left(_{c_{i} \in C L A U S E} \text { clause }_{i}\right) \wedge\left(\bigwedge_{v_{i} \in V A R} \neg v_{i}^{u}\right)\right) \supset \text { sat } \mid\right. \\
& \text { where } \text { clause }_{i} \text { is a formula } \\
& \text { obtained by replacing all occurrences of } \\
& \left.v_{i} \text { and } \neg v_{i} \text { in } c_{i} \text { with } v_{i}^{t} \text { and } v_{i}^{f} \text { respectively }\right\} \\
\mathcal{P}= & \{\mathbf{K} \text { sat } \leftarrow \mathbf{K} \text { sat }\} \cup \bigcup\left\{\left\{\left(\mathbf{K} v_{i}^{t}, \mathbf{K} v_{i}^{f} \leftarrow\right)\right\} \mid v_{i} \in V A R\right\}
\end{aligned}
$$

Figure 1: A disjunctive MKNF knowledge base

The entailment relation $\mathrm{OB}_{\mathcal{O}, S} \models v_{i}^{t}\left(\right.$ resp. $\mathrm{OB}_{\mathcal{O}, S} \models v_{i}^{f}$ ) holds if and only if $v_{i}^{t} \in S$ (resp. $v_{i}^{f} \in S$ ). What remains to show is that $\mathrm{OB}_{\mathcal{O}, S} \models$ sat can be checked in polynomial time when $\mathbf{K}$ sat $\notin S$. We call a set of $\mathbf{K}$-atoms $S$ consistent if it does not contain both $\mathbf{K} v_{i}^{t}$ and $\mathbf{K} v_{i}^{f}$ for every variable $v_{i} \in V A R$. If $S$ is not consistent, then we have $\mathrm{OB}_{\mathcal{O}, S} \models$ sat due to the second set of formulas in $\mathcal{O}$. We assume that $S$ is consistent. We call a set of $\mathbf{K}$-atoms $S$ total if it contains either $\mathbf{K} v_{i}^{t}$ or $\mathbf{K} v_{i}^{f}$ for each variable $v_{i} \in V A R$. We consider the cases where $S$ is total and where $S$ is not total. If $S$ is not total, we can construct a consistent first-order interpretation of $S \cup \pi(\mathcal{O})$ such that $v_{i}^{u}$ is true for some $v_{i} \in V A R$, thus $\mathrm{OB}_{\mathcal{O}, S} \not \mid$ sat if $S$ is consistent and not total. Now we assume that $S$ is total and it follows that $\bigwedge_{v_{i} \in V A R} \neg v_{i}^{u}$ is satisfied in the third set of formulas in $\mathcal{O}$. We refer to a model $M$ of $S \cup \pi(\mathcal{O})$ as a proper model if for every $v_{i} \in V A R$ we have $v_{i}^{f}$ (resp. $v_{i}^{t}$ ) to be false in $M$ if $v_{i}^{f} \notin S$ (resp. $v_{i}^{f} \notin S$ ). Observe that for all models of $S \cup \pi(\mathcal{O})$ modulo proper models, sat is true because of the second set of formulas in $\mathcal{O}$ (recall that $S$ is total and consistent). Note that for each proper model $M$ we have $M \models v_{i}^{f} \oplus v_{i}^{t}$ (where $\oplus$ is exclusive-or) because $S$ is consistent. The only case where $\mathrm{OB}_{\mathcal{O}, S} \not \models$ sat is if we have $\mathrm{OB}_{\mathcal{O}, S}=$ sat if and only if $S$ satisfies every formula clause $_{i}$. This can easily be checked in polynomial time.

(B) When determining whether the $\mathbf{K}$-atom $\mathbf{K}$ sat is unfounded w.r.t. $(\emptyset, \emptyset)$, we must consider each way to select a head-cut $R$. This part of the proof carries out almost identically to part 2 of our proof of Proposition 3 . We only outline the key differences: Rather than relying on head $(R) \cup \pi(\mathcal{O})$ to be inconsistent if $R$ does not correspond to a satisfying assignment of $S A T$ like in our proof of Proposition 3, we rely on there being a single model of head $(R) \cup \pi(\mathcal{O})$ where sat is false (See part (A) of this proof for details on proper models). This is enough to show that head $(R) \cup \mathrm{OB}_{\mathcal{O}, \emptyset} \not \neq$ sat. When only considering proper models of $\operatorname{head}(R) \cup \pi(\mathcal{O})$, we can ignore the second set of formulas in $\mathcal{O}$ because a set of rule atom pairs $R$ containing both $\left(r, v_{i}^{f}\right)$ and $\left(r, v_{i}^{t}\right)$ is not a valid head-cut. In order to determine whether a $\mathbf{K}$-atom
$\mathbf{K} a$ is unfounded w.r.t. $(\emptyset, \emptyset)$, we must exhaustively check head $(R) \cup \pi(\mathcal{O})$ for every head-cut $R$ and can conclude that $S A T$ is unsatisfiable. If we know that $S A T$ is unsatisfiable, there cannot exist a head-cut $R$, which proves that $\mathbf{K} a$ is not an unfounded atom.

\section{A DPLL-Based Solver}

In this section we formulate a DPLL-based solver. First, we construct a well-founded operator $W_{\mathcal{K}}^{(T, F)}$ using the greatest unfounded set approximator from the previous section:

$$
\begin{aligned}
& T_{\mathcal{K}}^{(T, F)}(X, Y)= \\
&\left\{\mathbf{K} a \mid \text { where } \mathrm{OB}_{\mathcal{O}, T \cup X}=a \text { for some } \mathbf{K} a \in \mathrm{KA}(\mathcal{K})\right\} \cup \\
&\{\mathbf{K} a \mid \text { where head }(r) \backslash F=\{\mathbf{K} a\} \text { and } \\
&\text { body }(r) \sqsubseteq(T \cup X, F \cup Y) \text { for some } r \in \mathcal{P}\}) \\
& W_{\mathcal{K}}^{(T, F)}(X, Y)=\left(T_{\mathcal{K}}^{(T, F)}(X, Y) \cup T\right. \\
&\left.\left(\operatorname{KA}(\mathcal{K}) \backslash Z_{\mathcal{K}}^{(T, F)}(X, Y)\right) \cup F\right)
\end{aligned}
$$

We show that this operator maintains the property shown in Proposition 2.

Proposition 6. If a dependable partition $(T, F)$ can be extended to an MKNF model $M$, then the dependable partition lfp $\left(W_{\mathcal{K}}^{(T, F)}\right)$ can also be extended to $M$.

Proof. It follows from Corollary 2 that if $(T, F)$ can be extended an MKNF model $M$, then $\left(T, F \cup Z_{\mathcal{K}}^{(T, F)}(T, F)\right)$ can be extended to $M$. What's left to show is that if $(T, F)$ can be extended to an MKNF model $M$, then $(T \cup$ $\left.\left.T_{\mathcal{K}}^{(T, F)}(T, F)\right), F\right)$ can be extended to $M$. Suppose that there is some $\mathbf{K}$-atom $\mathbf{K} a$ in $T \cap T_{\mathcal{K}}^{(T, F)}(T, F)$ ) such that $M \not \nvdash_{M K N F} \mathbf{K} a$. Then we either have that $\mathrm{OB}_{\mathcal{O}, T} \models a$, and thus $M \nvdash_{M K N F} \pi(\mathcal{O})$ or that $M \forall_{M K N F} \mathbf{K} h$ for each $\mathbf{K} h \in \operatorname{head}(r)$ and thus $M \forall_{M K N F} \pi(\mathcal{P})$. Either case contradicts the assumption that $M$ is an MKNF model of $\mathcal{K}$.

Following Ji et al. (2017), we construct an abstract solver in Algorithm 1 that prunes the search space for solving by using the $W_{\mathcal{K}}^{(T, F)}$ operator. The CHECK-MODEL procedure checks whether the MKNF interpretation

$$
\begin{aligned}
& \{I \mid \text { where } I \models \pi(\mathcal{O}), I \models t \\
& \quad \text { for each } \mathbf{K} t \in T \text {, and } I \not \models f \text { for each } \mathbf{K} f \in F\}
\end{aligned}
$$

is an MKNF model of $\mathcal{K}$ whenever the solver reaches a total dependable partition. This procedure is analogous to the NP-oracle required to check a model of a disjunctive logic program (Ben-Eliyahu and Dechter 1994). Further developments are required for a more precise definition of this procedure.

Proposition 7. Given a partial partition $(T, F)$ of $\mathrm{KA}(\mathcal{K})$, the invocation of Algorithm 1 solver $(\mathcal{K},(\emptyset, \emptyset))$ will return true if $(T, F)$ can be extended to an MKNF model of $\mathcal{K}$. 


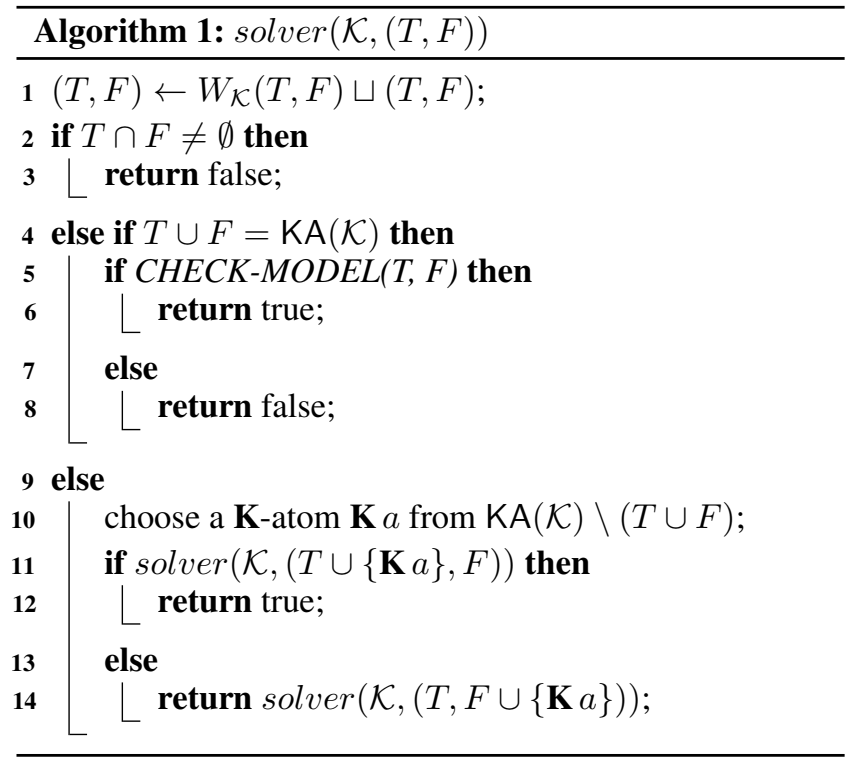

Proof. It follows from Proposition 6 that the extension of $(T, F)$ on the first line of the algorithm, $(T, F) \leftarrow$ $W_{\mathcal{K}}(T, F) \sqcup(T, F)$, does not miss any models. A model that induces a partition $(T, F)$ s.t. $T \cap F \neq \emptyset$ does not exist. Without the use of the $W_{\mathcal{K}}(T, F)$ operator, the solver algorithm will explore every partition $(T, F) \subseteq \mathrm{KA}(\mathcal{K}) \times \mathrm{KA}(\mathcal{K})$ where $T \cap F=\emptyset$. Thus, the usage of the $W_{\mathcal{K}}(T, F)$ operator simply prunes the search space.

Given Proposition 7, it is easy to modify Algorithm 1 to report models instead of returning a boolean value.

We have identified some fundamental challenges in computing unfounded sets for hybrid MKNF knowledge bases that make the problem intractable. The operator constructed by Ji et al. (2017) computes a subset of the greatest unfounded set and we build on this approximation with an extension for programs with rules with disjunctive heads.

\section{Related Work}

Ji et al. establish a definition of unfounded sets for normal hybrid MKNF knowledge bases and construct well-founded operators that can be directly embedded in a solver (2017). We extend their work by introducing a definition of unfounded sets that handles disjunctive rules, rules that have multiple K-atoms in their heads. Our extension borrows from the unfounded-set techniques outlined by Leone et al. (1997) for disjunctive logic programs but with a few noteworthy differences. Namely, our definition cannot be used directly for model-checking. If the ontology in $\mathcal{K}$ is empty, our definition is equivalent to Leon et al.'s for unfoundedfree partitions. Similarly, if $\mathcal{K}$ is a normal knowledge base, our definition is equivalent to Ji et al.'s definition.

Both Ji et al. and Leone et al. outline abstract solvers for finding models of their respective languages. These solvers follow the DPLL paradigm of exploring the search space for a model. Both solvers substantially prune their search space using unfounded sets. Because the complexity of modelchecking a disjunctive hybrid MKNF knowledge base is greater than that of normal hybrid MKNF knowledge bases (Motik and Rosati 2010), our abstract solver in this work consults a model checker once it reaches a total partition. This differs from the solver described by Ji et al. which does not rely on a model checker (2017). Leone et al.'s solver does not deepen its search on partial interpretations that assign unfounded atoms as true (partitions that cannot be extended to models) (1997). This aggressive pruning strategy requires, at each step of the solver, an invocation of an algorithm with a complexity of $\Delta_{2}^{P}[O(\log n)]$ (Leone, Rullo, and Scarcello 1997). Industry-grade solvers, such as Clingo (Gebser, Kaufmann, and Schaub 2012) or HEX (Eiter et al. . 2005), recognize the impracticality of enumerating all unfounded sets many times during the solving process and these solvers introduce approximations techniques. As a caveat of using approximations of unfounded sets, a solver may deepen its search on partial interpretations that cannot be extended to models. Because we rely on approximations of greatest unfounded sets, we think it is reasonable for our solver to employ similar strategies used by practical solvers and include some partitions that cannot be extended to models in its search.

Both Clingo and HEX have additional support for external atoms, atoms whose truth is dependant on external sources. Clingo 5 defines $\mathbb{T}$-stable semantics (Gebser et al. . 2016) to reason about external atoms via external theories. HEX defines semantics for external atoms using boolean functions that take a total interpretation as input (Eiter and Gottlob 1995). For any hybrid MKNF knowledge base, models of the accompanying ontology must be monotonic (Motik and Rosati 2010). While it may be possible to encode the semantics of hybrid MKNF knowledge bases using either the HEX or Clingo extensions, neither solution exploits the monotonicity of external sources and both support nonmonotonic models of the external theories.

\section{Conclusion}

We have provided a definition of unfounded sets for disjunctive hybrid MKNF knowledge bases, studied its properties, and formulated an operator to compute a subset of the greatest unfounded set of a knowledge base. This leads to a DPLL-based solver where after each decision constraint propagation is carried out by computing additional true and false atoms on top of the current partial partition. Our methods can be directly embedded into a solver for a drastic increase in efficiency when compared to a guess-and-verify solver, the current state of art for reasoning with disjunctive hybrid MKNF knowledge bases. The addition of ontologies to answer set programs brings new challenges, namely, there is a complexity increase in computing unfounded sets even in the case of normal hybrid MKNF knowledge bases. We leave computing unfounded sets in light of inconsistencies that arise because of $\mathcal{O}$ to future work. 


\section{References}

Ben-Eliyahu, R., and Dechter, R. 1994. Propositional semantics for disjunctive logic programs. Annals of Mathematics and Artificial Intelligence 12(1):53-87.

Eiter, T., and Gottlob, G. 1995. On the computational cost of disjunctive logic programming: Propositional case. Annals of Mathematics and Artificial Intelligence 15(3):289-323.

Eiter, T.; Ianni, G.; Schindlauer, R.; and Tompits, H. 2005. A uniform integration of higher-order reasoning and external evaluations in answer-set programming. 7.

Gebser, M.; Kaminski, R.; Kaufmann, B.; Ostrowski, M.; Schaub, T.; and Wanko, P. 2016. Theory solving made easy with clingo 5. In Carro, M.; King, A.; Saeedloei, N.; and Vos, M. D., eds., Technical Communications of the 32nd International Conference on Logic Programming, ICLP 2016 TCs, October 16-21, 2016, New York City, USA, volume 52 of OASICS, 2:1-2:15. Schloss Dagstuhl - Leibniz-Zentrum für Informatik.

Gebser, M.; Kaufmann, B.; and Schaub, T. 2012. Conflictdriven answer set solving: From theory to practice. Artificial Intelligence 187-188:52-89.

Ji, J.; Liu, F.; and You, J.-H. 2017. Well-founded operators for normal hybrid MKNF knowledge bases. Theory Pract. Log. Program. 17(5-6):889-905.

Leone, N.; Rullo, P.; and Scarcello, F. 1997. Disjunctive stable models: Unfounded sets, fixpoint semantics, and computation. Information and Computation 135(2):69-112.

Lifschitz, V. 1991. Nonmonotonic databases and epistemic queries. In Mylopoulos, J., and Reiter, R., eds., Proceedings of the 12th International Joint Conference on Artificial Intelligence. Sydney, Australia, August 24-30, 1991, 381-386. Morgan Kaufmann.

Motik, B., and Rosati, R. 2010. Reconciling description logics and rules. Journal of the ACM 57(5):1-62.

Nieuwenhuis, R.; Oliveras, A.; and Tinelli, C. 2006. Solving SAT and SAT Modulo Theories: From an abstract davisputnam-logemann-loveland procedure to DPLL(T). Journal of the ACM 53(6):937-977.

Sipser, M. 1996. Introduction to the Theory of Computation. International Thomson Publishing, first edition edition.

Van Gelder, A.; Ross, K. A.; and Schlipf, J. S. 1991. The well-founded semantics for general logic programs. Journal of the ACM 38(3):619-649. 